To cite: Bernatsky S, Velásquez García HA, Spinelli JJ, et al. Lupusrelated single nucleotide polymorphisms and risk of diffuse large B-cell lymphoma. Lupus Science \& Medicine 2017:4:e000187. doi:10.1136/lupus-2016000187

\section{Lupus-related single nucleotide polymorphisms and risk of diffuse large B-cell lymphoma}

Sasha Bernatsky, ${ }^{1}$ Héctor A Velásquez García, ${ }^{2}$ John J Spinelli, ${ }^{2}$ Patrick Gaffney, ${ }^{3}$ Karin E Smedby, ${ }^{4}$ Rosalind Ramsey-Goldman, ${ }^{5}$ Sophia S Wang, ${ }^{6}$ Hans-Olov Adami, ${ }^{7,8}$ Demetrius Albanes, ${ }^{9}$ Emanuele Angelucci, ${ }^{10}$ Stephen M Ansell, ${ }^{11}$ Yan W Asmann, ${ }^{12}$ Nikolaus Becker, ${ }^{13}$ Yolanda Benavente, ${ }^{14}$ Sonja I Berndt, ${ }^{9}$ Kimberly A Bertrand, ${ }^{15}$ Brenda M Birmann, ${ }^{16}$ Heiner Boeing, ${ }^{17}$ Paolo Boffetta, ${ }^{18}$ Paige M Bracci, ${ }^{19}$ Paul Brennan, ${ }^{20}$ Angela R Brooks-Wilson, ${ }^{21}$ James R Cerhan, ${ }^{22}$ Stephen J Chanock, ${ }^{9}$ Jacqueline Clavel, ${ }^{23}$ Lucia Conde, ${ }^{24}$ Karen H Cotenbader, ${ }^{25}$ David G Cox, ${ }^{26}$ Wendy Cozen, ${ }^{27}$ Simon Crouch, ${ }^{28}$ Anneclaire J De Roos, ${ }^{29}$ Silvia de Sanjose, ${ }^{14,30}$ Simonetta Di Lollo, ${ }^{31}$ W Ryan Diver, ${ }^{32}$ Ahmet Dogan, ${ }^{33}$ Lenka Foretova, ${ }^{34}$ Hervé Ghesquières, ${ }^{35}$ Graham G Giles, ${ }^{36,37}$ Bengt Glimelius, ${ }^{38}$ Thomas M Habermann, ${ }^{39}$ Corinne Haioun, ${ }^{40}$ Patricia Hartge, ${ }^{9}$ Henrik Hjalgrim, ${ }^{41}$ Theodore R Holford, ${ }^{42}$ Elizabeth A Holly, ${ }^{19}$ Rebecca D Jackson, ${ }^{43}$ Rudolph Kaaks, ${ }^{13}$ Eleanor Kane, ${ }^{28}$ Rachel S Kelly, ${ }^{16}$ Robert J Klein, ${ }^{44}$ Peter Kraft, ${ }^{8}$ Anne Kricker, ${ }^{45}$ Qing Lan, ${ }^{9}$ Charles Lawrence, ${ }^{46}$ Mark Liebow, ${ }^{11}$ Tracy Lightfoot, ${ }^{28}$ Brian K Link, ${ }^{47}$ Marc Maynadie, ${ }^{48}$ James McKay, ${ }^{20}$ Mads Melbye, ${ }^{41}$ Thierry J Molina, ${ }^{49}$ Alain Monnereau, ${ }^{23}$ Lindsay M Morton, ${ }^{9}$ Alexandra Nieters, ${ }^{50}$ Kari E North, ${ }^{51}$ Anne J Novak, ${ }^{11}$ Kenneth Offit, ${ }^{52}$ Mark P Purdue, ${ }^{53}$ Marco Rais, ${ }^{54}$ Jacques Riby, ${ }^{24}$ Eve Roman, ${ }^{28}$ Nathaniel Rothman, ${ }^{9}$ Gilles Salles, ${ }^{55}$ Gianluca Severi, ${ }^{56}$ Richard K Severson, ${ }^{57}$ Christine F Skibola, ${ }^{24}$ Susan L Slager, ${ }^{22}$ Alex Smith, ${ }^{28}$ Martyn T Smith, ${ }^{58}$ Melissa C Southey, ${ }^{59}$ Anthony Staines, ${ }^{60}$ Lauren R Teras, ${ }^{32}$ Carrie A Thompson, ${ }^{11}$ Hervé Tilly, ${ }^{61}$ Lesley F Tinker, ${ }^{62}$ Anne Tjonneland, ${ }^{63}$ Jenny Turner, ${ }^{64}$ Claire M Vajdic, ${ }^{65}$ Roel C H Vermeulen, ${ }^{66}$ Joseph Vijai, ${ }^{52}$ Paolo Vineis, ${ }^{67}$ Jarmo Virtamo, ${ }^{68}$ Zhaoming Wang, $^{69}$ Stephanie Weinstein, ${ }^{9}$ Thomas E Witzig, ${ }^{11}$ Andrew Zelenetz, ${ }^{52}$ Anne Zeleniuch-Jacquotte, ${ }^{70}$ Yawei Zhang, ${ }^{71}$ Tongzhang Zheng, ${ }^{72}$ Mariagrazia Zucca, ${ }^{73}$ Ann E Clarke ${ }^{74}$

- Additional material is available. To view please visit the journal online (http://dx. doi.org/10.1136/lupus-2016000187).

Received 29 September 2016 Revised 18 November 2016 Accepted 30 November 2016

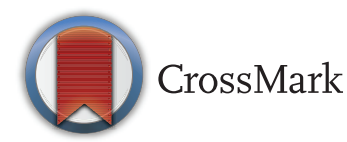

For numbered affiliations see end of article.

Correspondence to Dr Sasha Bernatsky; Sasha.bernatsky@mcgill.ca

\section{ABSTRACT}

Objective: Determinants of the increased risk of diffuse large B-cell lymphoma (DLBCL) in SLE are unclear. Using data from a recent lymphoma genomewide association study (GWAS), we assessed whether certain lupus-related single nucleotide polymorphisms (SNPS) were also associated with DLBCL.

Methods: GWAS data on European Caucasians from the International Lymphoma Epidemiology Consortium (InterLymph) provided a total of 3857 DLBCL cases and 7666 general-population controls. Data were pooled in a random-effects meta-analysis.

Results: Among the 28 SLE-related SNPs investigated, the two most convincingly associated with risk of DLBCL included the CD40 SLE risk allele rs4810485 on chromosome 20q13 (OR per risk allele $=1.09,95 \% \mathrm{Cl} 1.02$ to $1.16, \mathrm{p}=0.0134)$, and the HLA SLE risk allele rs 1270942 on chromosome 6 p21.33 (OR per risk allele $=1.17,95 \% \mathrm{Cl} 1.01$ to 1.36 , $p=0.0362$ ). Of additional possible interest were rs2205960 and rs12537284. The rs2205960 SNP, related to a cytokine of the tumour necrosis factor superfamily TNFSF4, was associated with an OR per risk allele of $1.07,95 \% \mathrm{Cl} 1.00$ to $1.16, p=0.0549$. The OR for the rs12537284 (chromosome 7q32, IRF5 gene) risk allele was $1.08,95 \% \mathrm{Cl} 0.99$ to 1.18 , $\mathrm{p}=0.0765$.

Conclusions: These data suggest several plausible genetic links between DLBCL and SLE.

Several recent studies have highlighted an increased risk of haematological malignancies, particularly non-Hodgkin's lymphoma (NHL), in patients with SLE. ${ }^{2}$ The determinants of the increased risk of NHL in SLE are unclear. The most common type of NHL in SLE (as in the general population) is the diffuse large B-cell lymphoma (DLBCL) 
subtype. Using data from a recent NHL genome-wide association study (GWAS), ${ }^{3}$ our objective was to determine if certain SLE-related single nucleotide polymorphisms (SNPs) were also associated with the risk of DLBCL.

We focused on 28 SNPs independently associated with SLE in European Caucasians. ${ }^{4}$ All of these SNPs have been strongly associated with lupus risk, with a $p$ value of $1 \times 10^{-7}$ or stronger. Our hypothesis was that these SNPs would also be associated with DLBCL risk.

\section{METHODS}

GWAS data on European Caucasians from the International Lymphoma Epidemiology Consortium (InterLymph http://www.epi.grants.cancer.gov/Inter Lymph) studies and participating cohort studies were based on a total of 3857 DLBCL cases and 7666 controls. Each participating study's investigators obtained approval from human subjects review committees and informed consent from all participants. De-identified data were provided by the InterLymph Data Coordinating Center (Mayo Clinic, Rochester, Minnesota, USA).
For each SLE-related SNP, the ORs and 95\% CIs were computed using a log-additive logistic regression model. Results from three previously conducted DLBCL GWAS studies were pooled in a random-effects meta-analysis. With 28 comparisons, an $\alpha$ of 0.05 would correspond to a Bonferroni-corrected $\mathrm{p}$ value of 0.0018 .

\section{RESULTS}

Among the 28 SLE-related SNPs investigated (table 1), the two most convincingly associated with risk of DLBCL when correcting for multiple comparisons included the CD40 SLE risk allele rs4810485 on chromosome 20q13 (OR per risk allele $=1.09,95 \%$ CI 1.02 to $1.16, p=0.0134$ ) and the HLA SLE risk allele rs1270942 on chromosome 6 p21.33 (OR per risk allele $1.17,95 \%$ CI 1.01 to 1.36 , $\mathrm{p}=0.0362$ ). Two other SNPs were of additional possible interest in DLBCL, with $95 \%$ CIs that just barely included the null value. The rs2205960 SNP, related to a cytokine of the tumour necrosis factor superfamily TNFSF4, was associated with an OR per risk allele of $1.07,95 \%$ CI 1.00 to $1.16, p=0.0549$. The OR for the SLE interferon regulatory factor (IRF5) risk allele

Table 1 SLE-related single nucleotide polymorphisms (SNPs) and ORs for diffuse large B-cell lymphoma (DLBCL) in European Caucasians in InterLymph data

\begin{tabular}{|c|c|c|c|c|c|c|c|}
\hline \multirow{2}{*}{$\frac{\text { Gene }}{C D 40}$} & \multirow{2}{*}{$\begin{array}{l}\text { Chromosome } \\
20\end{array}$} & \multirow{2}{*}{$\begin{array}{l}\text { SNP } \\
\text { rs4810485 }\end{array}$} & \multicolumn{2}{|c|}{$\begin{array}{l}\text { Allele }^{\star} \\
\text { DLBCL SLE } \\
\text { ref. }\end{array}$} & \multirow{2}{*}{$\begin{array}{l}\text { DLBCL } \\
\text { OR } \\
\text { C }\end{array}$} & \multirow{2}{*}{$\begin{array}{l}\text { DLBCL } 95 \% \text { Cl } \\
1.088(1.017 \text { to } 1.162)\end{array}$} & \multirow{2}{*}{$\begin{array}{l}\text { p Value* } \\
\text { DLBCL }\end{array}$} \\
\hline & & & $\mathrm{T}$ & $\mathrm{T}$ & & & \\
\hline$H L A$ & 6 & rs1270942 & G & G & A & 1.171 (1.010 to 1.357$)$ & 0.036172 \\
\hline TNFSF4 & 1 & rs2205960 & A & A & G & 1.074 (0.998 to 1.156$)$ & 0.054899 \\
\hline IRF5 & 7 & rs12537284 & A & A & G & 1.081 (0.992 to 1.179$)$ & 0.076450 \\
\hline IL/10 & 1 & rs3024505 & A & A & G & $1.102(0.898$ to 1.353$)$ & 0.352319 \\
\hline BANK1 & 4 & rs10516487 & A & A & G & 1.035 (0.969 to 1.106$)$ & 0.303231 \\
\hline Mir146a & 5 & rs57095329 & G & $\mathrm{G}$ & A & $1.020(0.756$ to 1.377$)$ & 0.896089 \\
\hline ITGAM & 16 & rs9888739 & $\mathrm{T}$ & $\mathrm{T}$ & C & 1.008 (0.923 to 1.102$)$ & 0.851519 \\
\hline IFIH1 & 2 & rs1990760 & $\mathrm{T}$ & $\mathrm{T}$ & C & 1.037 (0.978 to 1.101$)$ & 0.223359 \\
\hline TNFAIP3 & 6 & rs77449323 & A & A & G & 1.053 (0.884 to 1.253$)$ & 0.564425 \\
\hline NCF2 & 1 & rs17849502 & $\mathrm{T}$ & $\mathrm{G}$ & G & $1.050(0.892$ to 1.236$)$ & 0.554699 \\
\hline STAT4 & 2 & rs7582694 & G & C & C & $1.110(0.977$ to 1.260$)$ & 0.108048 \\
\hline PTPN22 & 1 & rs2476601 & $\mathrm{G}$ & A & A & 1.043 (0.937 to 1.161 & 0.441704 \\
\hline TYK2 & 19 & rs280519 & G & A & A & 1.016 (0.959 to 1.077$)$ & 0.582604 \\
\hline PHRF1/IRF7/ & 11 & rs4963128 & $\mathrm{C}$ & $\mathrm{T}$ & $\mathrm{T}$ & 1.018 (0.956 to 1.085$)$ & 0.570646 \\
\hline \multicolumn{8}{|l|}{ KIAA1542 } \\
\hline$C D 44$ & 11 & rs507230 & A & G & G & 1.000 (0.941 to 1.062$)$ & 0.987988 \\
\hline XKR6 & 8 & rs6985109 & A & G & G & 1.040 (0.981 to 1.103$)$ & 0.187826 \\
\hline JAZF1 & 7 & rs849142 & C & $\mathrm{T}$ & $\mathrm{T}$ & $1.012(0.903$ to 1.134$)$ & 0.836267 \\
\hline UBE2L3 & 22 & rs463426 & C & G & $\mathrm{T}$ & 1.060 (0.938 to 1.197$)$ & 0.349982 \\
\hline$B L K$ & 8 & rs7812879 & C & A & $\mathrm{T}$ & 1.058 (0.956 to 1.172$)$ & 0.276113 \\
\hline FCGR2A, FCGR3B & 1 & rs1801274 & G & $\mathrm{T}$ & A & $1.023(0.913$ to 1.147$)$ & 0.693045 \\
\hline$I K Z F 1$ & 7 & rs4917014 & G & C & $\mathrm{T}$ & $1.020(0.916$ to 1.138$)$ & 0.710394 \\
\hline$L Y N$ & 8 & rs7829816 & G & C & A & 1.031 (0.959 to 1.107$)$ & 0.411987 \\
\hline TNIP1 & 5 & rs10036748 & $\mathrm{T}$ & G & C & 1.015 (0.950 to 1.085$)$ & 0.652213 \\
\hline IRF8 & 16 & rs2280381 & $\mathrm{T}$ & A & C & 1.096 (0.933 to 1.287$)$ & 0.265341 \\
\hline ATG5 & 6 & rs548234 & $\mathrm{T}$ & G & C & $1.033(0.936$ to 1.140$)$ & 0.518828 \\
\hline$P X K$ & 3 & rs6445975 & $\mathrm{T}$ & C & G & 1.011 (0.945 to 1.083$)$ & 0.743076 \\
\hline IL2/IL21 & 4 & rs907715 & $\mathrm{T}$ & $\mathrm{G}$ & C & 1.033 (0.967 to 1.104$)$ & 0.339144 \\
\hline
\end{tabular}


rs12537284 (chromosome 7q32, gene) was $1.08,95 \%$ CI 0.99 to $1.18, \mathrm{p}=0.0765$. A table presenting the studyspecific contributions to the meta-analysis is provided in the online supplemental material.

\section{DISCUSSION}

Multiple studies have highlighted an increased risk of haematological malignancies, particularly NHL, in patients with SLE. To date, the reason for this excess risk has remained elusive. Recently, advances have been made in our understanding of lymphoma risk in other autoimmune rheumatic diseases, such as primary Sjögren's syndrome, where the majority of patients with mucosa-associated lymphoid tissue (MALT) lymphoma have either germline polymorphisms of TNFAIP3 related to the A20 protein important in nuclear factor $\mathrm{\kappa B}$ activation or somatic alterations of the gene within the lymphoma tissue. ${ }^{5}$ In their assessment of genetic risk overlap between rheumatoid arthritis (RA) and haematological cancers, Okada et $a t^{6}$ found that polymorphisms of TNFAIP3 were common to both RA and Hodgkin's lymphoma. Our analyses did not confirm a strong relationship with the lupus-related TNFAIP3 SNP rs7749323 specifically for DLBCL, but this may be a power issue, or may reflect the importance of different pathways for different haematological risk profiles across different autoimmune rheumatic diseases. Of note, our analyses were done in Caucasian populations; several non-Caucasian race/ethnic groups (eg, blacks, Asians) may have different genetic risk profiles and clinical presentations, thus future analyses could consider these populations as well. We have previously shown that the increased risk of lymphoma in SLE is similar across white, black and Asian patients. ${ }^{7}$ In addition, it may be that specific genetic risk factors for different clinical SLE manifestations may drive some of the risk of lymphoma, although we were unable to investigate that hypothesis here.

Existing data do suggest that some human leukocyte antigen (HLA) polymorphisms influence risk of DLBCL. ${ }^{8}$ In recent DLBCL GWAS analyses, HLA-B 08-01 reached genome-wide significance. ${ }^{4}$ In SLE, the strongest association in HLA is for the Class II allele DRB1*0301. This allele is in strong linkage disequilibrium with HLA-B*0801 in Caucasians so we are likely tagging the same HLA effect. ${ }^{9}$ CD 40, a member of the tumour necrosis superfamily, plays a central role in regulating immune cells; CD40 is expressed on several B-cell neoplasms including DLBCL. Data have suggested a possible role for functional polymorphisms (specifically, C vs T, rs1883832) in the TNFRSF5 gene encoding CD40 in lymphomas originating within the germinal centre (both DLBCL and follicular). ${ }^{10}$ Tumour necrosis factor ligand superfamily involvement has been suggested in the pathology of malignant lymphomas. ${ }^{11}$ Furthermore, in human NHL B-cell lines, IRF5 initiates a regulatory cascade by inducing the transcription factor activator protein 1 (AP-1) and cooperating with nuclear factor kappa B (NF-kB), which appears to represent a potentially important tumour promoting role of IRF5 in lymphoma. ${ }^{12}$

Not all of the excess risk of haematological malignancies in SLE is necessarily due to genetic factors; exposures within the environment may also be at play. However, in the InterLymph Subtypes pooling project, autoimmune diseases as a risk for lymphoma appeared to be independent of other potentially shared environmental risk factors (body mass index, sun, alcohol, occupation, etc). ${ }^{13}$ In the work of Ekström Smedby et al, SLE was associated with a 2.7-fold increase in risk of NHL risk overall; this was highest among patients with SLE of short duration (2-5 years), but a near twofold increase was also observed with more than 10 years of disease. Use of corticosteroid and immunosuppressive drugs categorically was not clearly linked to higher or lower risk, but analyses were not detailed. ${ }^{2}$ Two very comprehensive case-control studies of SLE-related medications have suggested a link between cyclophosphamide (used intravenously in severe or resistant forms of SLE, especially nephritis) and haematological malignancies in general ${ }^{14}$ (and specifically, in lymphoma ${ }^{15}$ ). Fortunately, lymphoma after cyclophosphamide SLE treatment is a relatively uncommon outcome. Future studies of interactions between genetic factors and drug exposures may be warranted.

In conclusion, we studied a large GWAS datasets and found several plausible pathways linking DLBCL and SLE. Given that cyclophosphamide exposure in SLE is also associated with DLBCL risk, future studies might be able to explore whether these genetic risk factors may aid in risk stratification and decision-making when cyclophosphamide treatment is being considered for severe forms of SLE.

\section{Author affiliations}

${ }^{1}$ Division of Clinical Epidemiology, Research Institute of the McGill University Health Centre, Montreal, Canada

${ }^{2} \mathrm{BC}$ Cancer Research Centre and School of Population and Public Health, University of British Columbia, Vancouver, Canada

${ }^{3}$ Arthritis and Clinical Immunology Research Program, Oklahoma Medical Research Foundation, Oklahoma City, Oklahoma, USA

${ }^{4}$ Clinical Epidemiology Unit, Department of Medicine, Karolinska Institutet, and Hematology Center, Karolinska University Hospital, Stockholm, Sweden

${ }^{5}$ Feinberg School of Medicine, Northwestern University, Chicago, USA

${ }^{6}$ Division of Cancer Etiology, Department of Population Sciences, Beckman Research Institute, Duarte, USA

${ }^{7}$ Department of Medical Epidemiology and Biostatistics, Karolinska Institutet, Stockholm, Sweden

${ }^{8}$ Department of Epidemiology, Harvard T.H. Chan School of Public Health, Boston, USA

${ }^{9}$ Division of Cancer Epidemiology and Genetics, National Cancer Institute, Bethesda, USA

${ }^{10}$ Hematology Unit, Ospedale Oncologico di Riferimento Regionale 'A. Businco', Cagliari, Italy

${ }^{11}$ Department of Medicine, Mayo Clinic, Rochester, USA

${ }^{12}$ Division of Biomedical Statistics and Informatics, Department of Health Sciences Research, Mayo Clinic, Jacksonville, USA

${ }^{13}$ Division of Cancer Epidemiology, German Cancer Research Center (DKFZ), Heidelberg, Germany

${ }^{14}$ Cancer Epidemiology Research Programme, Catalan Institute of OncologyIDIBELL, L'Hospitalet de Llobregat, Barcelona, Spain 
${ }^{15}$ Slone Epidemiology Center, Boston University, Boston, USA

${ }^{16}$ Channing Division of Network Medicine, Department of Medicine, Brigham and Women's Hospital and Harvard Medical School, Boston, USA

${ }^{17}$ Department of Epidemiology, German Institute for Human Nutrition, Potsdam, Germany

${ }^{18}$ The Tisch Cancer Institute, Icahn School of Medicine at Mount Sinai, New York, USA

${ }^{19}$ Department of Epidemiology and Biostatistics, University of California

San Francisco, San Francisco, USA

${ }^{20}$ International Agency for Research on Cancer (IARC), Lyon, France

${ }^{21}$ Genome Sciences Centre, BC Cancer Agency, Vancouver, Canada

${ }^{22}$ Department of Health Sciences Research, Mayo Clinic, Rochester, USA

${ }^{23}$ Epidemiology of childhood and adolescent cancers Group, Inserm, Center of

Research in Epidemiology and Statistics Sorbonne Paris Cité (CRESS), Paris, France

${ }^{24}$ Department of Epidemiology, School of Public Health and Comprehensive Cancer Center, University of Alabama at Birmingham, Birmingham, USA

${ }^{25}$ Division of Rheumatology, Immunology and Allergy, Brigham and Women's Hospital, Boston, USA

${ }^{26}$ INSERM U1052, Cancer Research Center of Lyon, Centre Léon Bérard, Lyon, France

${ }^{27}$ Department of Preventive Medicine, USC Keck School of Medicine,

University of Southern California, Los Angeles, USA

${ }^{28}$ Department of Health Sciences, University of York, York, UK

${ }^{29}$ Department of Environmental and Occupational Health, Dornsife School of Public Health at Drexel University, Philadelphia, USA

${ }^{30}$ CIBER de Epidemiología y Salud Pública (CIBERESP), Barcelona, Spain

${ }^{31}$ Department of Surgery and Translational Medicine, Section of Anatomo-

Pathology, University of Florence, Florence, Italy

${ }^{32}$ Epidemiology Research Program, American Cancer Society, Atlanta, USA

${ }^{33}$ Departments of Laboratory Medicine and Pathology, Memorial Sloan

Kettering Cancer Center, New York, USA

${ }^{34}$ Department of Cancer Epidemiology and Genetics, Masaryk Memorial Cancer Institute and MF MU, Brno, Czech Republic

${ }^{35}$ Department of Hematology, Centre Léon Bérard, Lyon, France

${ }^{36}$ Cancer Epidemiology Centre, Cancer Council Victoria, Melbourne, Australia

${ }^{37}$ Centre for Epidemiology and Biostatistics, Melbourne School of Population and Global Health, University of Melbourne, Melbourne, Australia

${ }^{38}$ Department of Immunology, Genetics and Pathology, Uppsala University,

Uppsala, Sweden

${ }^{39}$ Division of Hematology, Department of Medicine, Mayo Clinic, Rochester, USA

${ }^{40}$ Lymphoid Malignancies Unit, Henri Mondor Hospital and University Paris Est, Créteil, France

${ }^{41}$ Division of Health Surveillance and Research, Department of Epidemiology Research, Statens Serum Institut, Copenhagen, Denmark

${ }^{42}$ Department of Biostatistics, Yale School of Public Health, New Haven, USA

${ }^{43}$ Division of Endocrinology, Diabetes and Metabolism, The Ohio State

University, Columbus, USA

${ }^{44}$ Icahn Institute for Genomics and Multiscale Biology, Department of

Genetics and Genomic Sciences, Icahn School of Medicine at Mount Sinai, New York, USA

${ }^{45}$ Sydney School of Public Health, The University of Sydney, Sydney, Australia

${ }^{46}$ Westat Inc, Rockville, USA

${ }^{47}$ Department of Internal Medicine, Carver College of Medicine, The University of lowa, lowa City, USA

${ }^{48}$ Registre des Hémopathies Malignes de Côte d'Or, EA 4184, Univ.

Bourgogne Franche-Comté and Dijon University Hospital, Dijon, France

${ }^{49}$ Department of Pathology, AP-HP, Necker Enfants malades, Université Paris

Descartes, Sorbonne Paris Cité, France

${ }^{50}$ Center for Chronic Immunodeficiency, University Medical Center Freiburg, Freiburg, Germany

${ }^{51}$ Department of Epidemiology, University of North Carolina at Chapel Hill,

Chapel Hill, USA

${ }^{52}$ Department of Medicine, Memorial Sloan Kettering Cancer Center,

New York, USA

${ }^{53}$ Ontario Health Study, Toronto, Canada

${ }^{54}$ Department of Public Health, Clinical and Molecular Medicine, University of

Cagliari, Monserrato, Italy
${ }^{55}$ Department of Hematology, Hospices Civils de Lyon, Pierre benite Cedex, France

${ }^{56}$ Human Genetics Foundation, Turin, Italy

${ }^{57}$ Department of Family Medicine and Public Health Sciences, Wayne State University, Detroit, USA

${ }^{58}$ Division of Environmental Health Sciences, University of California Berkeley School of Public Health, Berkeley, USA

${ }^{59}$ Genetic Epidemiology Laboratory, Department of Pathology, University of

Melbourne, Melbourne, Australia

${ }^{60}$ School of Nursing and Human Sciences, Dublin City University, Dublin, Ireland

${ }^{61}$ Centre Heni Becquerel, Université de Rouen, Rouen, France

${ }^{62}$ Division of Public Health Sciences, Fred Hutchinson Cancer Research

Center, Seattle, USA

${ }^{63}$ Danish Cancer Society Research Center, Copenhagen, Denmark

${ }^{64}$ Faculty of Medicine and Health Sciences, Macquarie University, Sydney,

Australia

${ }^{65}$ Centre for Big Data Research in Health, University of New South Wales,

Sydney, Australia

${ }^{66}$ Institute for Risk Assessment Sciences, Utrecht University, Utrecht, The Netherlands

${ }^{67}$ MRC-PHE Centre for Environment and Health, School of Public Health, Imperial College London, London, UK

${ }^{68}$ Chronic Disease Prevention Unit, National Institute for Health and Welfare, Helsinki, Finland

${ }^{69}$ Department of Computational Biology, St. Jude Children's Research

Hospital, Memphis, Tennessee, USA

${ }^{70}$ Department of Population Health, New York University School of Medicine, New York, USA

${ }^{71}$ Department of Environmental Health Sciences, Yale School of Public Health, New Haven, USA

${ }^{72}$ Department of Epidemiology, Brown School of Public Health, Providence, USA

${ }^{73}$ Department of Biomedical Science, University of Cagliari, Monserrato, Italy

${ }^{74}$ Division of Rheumatology, University of Calgary, Calgary, Canada

Contributors All authors contributed to study design and/or data collection and/or analysis. All authors contributed to the manuscript and approve the final version.

Funding Support for the logistical needs of the InterLymph Consortium is provided by NCl's Division of Cancer Epidemiology and Genetics (DCEG), the Epidemiology and Genomics Research Program (EGRP) of the Division of Cancer Control and Population Sciences (DCCPS), the International Agency for Research on Cancer (IARC) and the Leukaemia Research Fund

\section{Support for individual studies}

ATBC: The $\alpha$-Tocopherol, $\beta$-Carotene Cancer Prevention Study is supported by the Intramural Research Program of the U.S. National Cancer Institute, National Institutes of Health and by U.S. Public Health Service contract HHSN261201500005C from the National Cancer Institute, Department of Health and Human Services.

BC: Canadian Institutes for Health Research (CIHR); Canadian Cancer Society; Michael Smith Foundation for Health Research.

CPS-II: The Cancer Prevention Study-II (CPS-II) Nutrition Cohort is supported by the American Cancer Society. Genotyping for all CPS-II samples were supported by the Intramural Research Program of the National Institutes of Health, NCl, Division of Cancer Epidemiology and Genetics. The authors would also like to acknowledge the contribution to this study from central cancer registries supported through the Centers for Disease Control and Prevention National Program of Cancer Registries and cancer registries supported by the National Cancer Institute Surveillance Epidemiology and End Results programme.

ELCCS: Bloodwise (formerly Leukaemia \& Lymphoma Research); grant reference 0073

ENGELA: Association pour la Recherche contre le Cancer (ARC), Institut National du Cancer (INCa), Fondation de France, Fondation contre la 
Leucémie, Agence nationale de sécurité sanitaire de l'alimentation, de l'environnement et du travail (ANSES).

EPIC: Coordinated Action (Contract \#006438, SP23-CT-2005-006438); HuGeF (Human Genetics Foundation), Torino, Italy; Cancer Research UK; Danish Cancer Society.

EpiLymph: European Commission (grant references QLK4-CT-2000-00422 and FOOD-CT-2006-023103); the Spanish Ministry of Health (grant references CIBERESP, PI11/01810, PI14/01219, RCESP C03/09, RTICESP C03/10 and RTIC RD06/0020/0095), the Marató de TV3 Foundation (grant reference 051210), the Agència de Gestiód'AjutsUniversitarisi de Recerca-Generalitat de Catalunya (grant reference 2014SRG756) who had no role in the data collection, analysis or interpretation of the results; the NIH (contract N01-CO-12400); the Compagnia di San Paolo-Programma Oncologia; the Federal Office for Radiation Protection grants StSch4261 and StSch4420, the José Carreras Leukemia Foundation grant DJCLS-R12/23, the German Federal Ministry for Education and Research (BMBF-01-E0-1303); the Health Research Board, Ireland and Cancer Research Ireland; Czech Republic supported by MH CZ-DRO (MMCl, 00209805) and by MEYS-NPS IL01413; Fondation de France and Association de Recherche Contre le Cancer.

GEC/Mayo GWAS: National Institutes of Health (CA118444, CA148690, CA92153). Intramural Research Program of the NIH, National Cancer Institute. Veterans Affairs Research Service. Data collection for Duke University was supported by a Leukemia \& Lymphoma Society Career Development Award the Bernstein Family Fund for Leukemia and Lymphoma Research and the National Institutes of Health (K08CA134919), National Center for Advancing Translational Science (UL1 TR000135).

HPFS: The HPFS was supported in part by National Institutes of Health grants CA167552, CA149445, CA098122 and CA098566. We would like to thank the participants and staff of the Health Professionals Follow-up Study for their valuable contributions as well as the following state cancer registries for their help: AL, AZ, AR, CA, CO, CT, DE, FL, GA, ID, IL, IN, IA, KY, LA, ME, MD, MA MI, NE, NH, NJ, NY, NC, ND, OH, OK, OR, PA, RI, SC, TN, TX, VA, WA, WY. The authors assume full responsibility for analyses and interpretation of these data

Iowa-Mayo SPORE: NCI Specialized Programs of Research Excellence (SPORE) in Human Cancer (P50 CA97274); National Cancer Institute (P30 CA086862, P30 CA15083); Henry J. Predolin Foundation.

Italian GxE: Italian Association for Cancer Research (AIRC, Investigator Grant 11855) (PC); Fondazione Banco di Sardegna 2010-2012 and Regione Autonoma della Sardegna (LR7 CRP-59812/2012) (MGE)

Mayo Clinic Case-Control: National Institutes of Health (R01 CA92153); National Cancer Institute (P30 CA015083)

MCCS: The Melbourne Collaborative Cohort Study recruitment was funded by VicHealth and Cancer Council Victoria. The MCCS was further supported by Australian NHMRC grants 209057, 251553 and 504711 and by infrastructure provided by Cancer Council Victoria. Cases and their vital status were ascertained through the Victorian Cancer Registry (VCR) and the Australian Institute of Health and Welfare (AIHW), including the National Death Index and the Australian Cancer Database.

MD Anderson: Institutional support to the Center for Translational and Public Health Genomics.

MSKCC: Geoffrey Beene Cancer Research Grant, Lymphoma Foundation (LF5541); Barbara K. Lipman Lymphoma Research Fund (74419); Robert and Kate Niehaus Clinical Cancer Genetics Research Initiative (57470); U01 HG007033; ENCODE; U01 HG007033.

NCI-SEER: Intramural Research Program of the National Cancer Institute, National Institutes of Health, and Public Health Service (N01-PC-65064, N01-PC-67008, N01-PC-67009, N01-PC-67010, N02-PC-71105). NHS-The NHS was supported in part by National Institutes of Health grants CA186107, CA87969, CA49449, CA149445, CA098122 and CA098566. We would like to thank the participants and staff of the Nurses' Health Study for their valuable contributions as well as the following state cancer registries for their help: $A L$, $\mathrm{AZ}, \mathrm{AR}, \mathrm{CA}, \mathrm{CO}, \mathrm{CT}, \mathrm{DE}, \mathrm{FL}, \mathrm{GA}, \mathrm{ID}, \mathrm{IL}$, IN, IA, KY, LA, ME, MD, MA, MI, NE, NH, NJ, NY, NC, ND, OH, OK, OR, PA, RI, SC, TN, TX, VA, WA, WY. The authors assume full responsibility for analyses and interpretation of these data.

NSW: NSW was supported by grants from the Australian National Health and Medical Research Council (ID990920), the Cancer Council NSW, and the University of Sydney Faculty of Medicine.

NYU-WHS: National Cancer Institute (UM1 CA182934, P30 CA016087); National Institute of Environmental Health Sciences (ES000260).

PLCO: This research was supported by the Intramural Research Program of the National Cancer Institute and by contracts from the Division of Cancer Prevention, National Cancer Institute, NIH, DHHS.

SCALE: Swedish Cancer Society (2009/659). Stockholm County Council (20110209) and the Strategic Research Program in Epidemiology at Karolinska Institute. Swedish Cancer Society grant (02 6661). National Institutes of Health (5R01 CA69669-02); Plan Denmark. UCSF-UCSF and the UCSF NHL study were supported by the following grants (including joint grants to University California Berkley (Skibola and M. Smith)): NIH grant R01-CA45614; NCl grant R01 CA87014; NCl grant R03 CA89745; NCl 263-MQ-701711; NCl grant R01 CA122663; NCI grant R01 CA104682; NCl grant P01-CA34233.

UCSF2: The UCSF studies were supported by the NCI, National Institutes of Health, CA1046282 and CA154643. The collection of cancer incidence data used in this study was supported by the California Department of Health Services as part of the state-wide cancer reporting programme mandated by California Health and Safety Code Section 103885; the National Cancer Institute's Surveillance, Epidemiology, and End Results Program under contract HHSN261201000140C awarded to the Cancer Prevention Institute of California, contract HHSN261201000035C awarded to the University of Southern California, and contract HHSN261201000034C awarded to the Public Health Institute; and the Centers for Disease Control and Prevention's National Program of Cancer Registries, under agreement \#1U58 DP000807-01 awarded to the Public Health Institute. The ideas and opinions expressed herein are those of the authors, and endorsement by the State of California, the California Department of Health Services, the National Cancer Institute, or the Centers for Disease Control and Prevention or their contractors and subcontractors is neither intended nor should be inferred.

UTAH/Sheffield: National Institutes of Health CA134674. Partial support for data collection at the Utah site was made possible by the Utah Population Database (UPDB) and the Utah Cancer Registry (UCR). Partial support for all datasets within the UPDB is provided by the Huntsman Cancer Institute $(\mathrm{HCl})$ and the $\mathrm{HCl}$ Cancer Center Support grant, P30 CA42014. The UCR is supported in part by NIH contract HHSN261201000026C from the National Cancer Institute SEER Program with additional support from the Utah State Department of Health and the University of Utah. Partial support for data collection in Sheffield, UK was made possible by funds from Yorkshire Cancer Research and the Sheffield Experimental Cancer Medicine Centre. We thank the NCRI Haemato-Oncology Clinical Studies Group, colleagues in the North Trent Cancer Network the North Trent Haemato-Oncology Database.

WHI: WHI investigators are: Program Office —(National Heart, Lung, and Blood Institute, Bethesda, Maryland) Jacques Rossouw, Shari Ludlam, Dale Burwen, Joan McGowan, Leslie Ford, and Nancy Geller; Clinical Coordinating Center-(Fred Hutchinson Cancer Research Center, Seattle, WA) Garnet Anderson, Ross Prentice, Andrea LaCroix, and Charles Kooperberg; Investigators and Academic Centers-(Brigham and Women's Hospital, Harvard Medical School, Boston, MA) JoAnn E. Manson; (MedStar Health Research Institute/Howard University, Washington, DC) Barbara V. Howard; (Stanford Prevention Research Center, Stanford, CA) Marcia L. Stefanick; (The Ohio State University, Columbus, $\mathrm{OH}$ ) Rebecca Jackson; (University of Arizona, Tucson/Phoenix, AZ) Cynthia A. Thomson; (University at Buffalo, Buffalo, NY) Jean Wactawski-Wende; (University of Florida, Gainesville/ Jacksonville, FL) Marian Limacher; (University of lowa, lowa City/Davenport, IA) Robert Wallace; (University of Pittsburgh, Pittsburgh, PA) Lewis Kuller; (Wake Forest University School of Medicine, Winston-Salem, NC) Sally Shumaker; Women's Health Initiative Memory Study—(Wake Forest University School of Medicine, Winston-Salem, NC) Sally Shumaker. The WHI program is funded by the National Heart, Lung, and Blood Institute, National Institutes of Health, U.S. Department of Health and Human Services through contracts 
HHSN268201100046C, HHSN268201100001C, HHSN268201100002C, HHSN268201100003C, HHSN268201100004C and HHSN271201100004C.

YALE: National Cancer Institute (CA62006); National Cancer Institute (CA165923)

Competing interests None declared.

Ethics approval Each participating study's investigators obtained approval from human subjects review committees and informed consent from all participants.

Provenance and peer review Not commissioned; externally peer reviewed.

Data sharing statement No additional data are available.

Open Access This is an Open Access article distributed in accordance with the Creative Commons Attribution Non Commercial (CC BY-NC 4.0) license, which permits others to distribute, remix, adapt, build upon this work noncommercially, and license their derivative works on different terms, provided the original work is properly cited and the use is non-commercial. See: http:// creativecommons.org/licenses/by-nc/4.0/

\section{REFERENCES}

1. Bernatsky S, Ramsey-Goldman R, Labrecque J, et al. Cancer risk in systemic lupus: an updated international multi-centre cohort study. $J$ Autoimmun 2013:42:130-5.

2. Ekström Smedby K, Vajdic CM, Falster M, et al. Autoimmune disorders and risk of non-Hodgkin lymphoma subtypes: a pooled analysis within the InterLymph Consortium. Blood 2008;111:4029-38.

3. Morton LM, Sampson JN, Cerhan JR, et al. Rationale and design of the international lymphoma epidemiology consortium (InterLymph) non-Hodgkin lymphoma subtypes project. J Natl Cancer InstMonogr 2014;2014:1-14.

4. Cui Y, Sheng Y, Zhang X. Genetic susceptibility to SLE: recent progress from GWAS. J Autoimmun 2013;41:25-33.
5. Nocturne G, Boudaoud S, Miceli-Richard C, et al. Germline and somatic genetic variations of TNFAIP3 in lymphoma complicating primary Sjögren's syndrome. Blood 2013;122:4068-76.

6. Okada Y, Wu D, Trynka G, et al. Genetics of rheumatoid arthritis contributes to biology and drug discovery. Nature 2014;506: 376-81.

7. Bernatsky S, Boivin JF, Joseph L, et al. Race/ethnicity and cancer occurrence in systemic lupus erythematosus. Arthritis Rheum 2005;53:781-4.

8. Alcoceba M, Sebastián E, Marín L, et al. HLA specificities are related to development and prognosis of diffuse large B-cell lymphoma. Blood 2013;122:1448-54.

9. Morris DL, Taylor KE, Fernando MM, et al. Unraveling multiple MHC gene associations with systemic lupus erythematosus: model choice indicates a role for HLA alleles and non-HLA genes in Europeans. Am J Hum Genet 2012;91:778-93.

10. Nieters A, Bracci PM, de Sanjosé S, et al. A functional TNFRSF5 polymorphism and risk of non-Hodgkin lymphoma, a pooled analysis. Int J Cancer 2011;128:1481-5.

11. Wang SS, Purdue MP, Cerhan JR, et al. Common gene variants in the tumor necrosis factor (TNF) and TNF receptor superfamilies and NF-kB transcription factors and non-Hodgkin lymphoma risk. PLOS ONE 2009;4:e5360.

12. Kreher S, Bouhlel MA, Cauchy P, et al. Mapping of transcription factor motifs in active chromatin identifies IRF5 as key regulator in classical Hodgkin lymphoma. Proc Natl Acad Sci USA 2014;111: E4513-22.

13. Wang SS, Flowers CR, Kadin ME, et al. Medical history, lifestyle, family history, and occupational risk factors for peripheral T-cell lymphomas: the InterLymph Non-Hodgkin Lymphoma Subtypes Project. J Natl Cancer Inst Monographs 2014;2014 66-75.

14. Bernatsky S, Joseph L, Boivin JF, et al. The relationship between cancer and medication exposures in systemic lupus erythaematosus: a case-cohort study. Ann Rheum Dis 2008;67:74-9.

15. Bernatsky S, Ramsey-Goldman R, Joseph L, et al. Lymphoma risk in systemic lupus: effects of disease activity versus treatment. Ann Rheum Dis 2014;73:138-42. 